\title{
Kirchhoff's Loop Law and the maximum entropy production principle
}

\author{
Paško Županovid* and Davor Juretiđ \\ Faculty of Natural Sciences, Mathematics and Education \\ University of Split, Teslina 12, 21000 Split \\ Croatia \\ Srećko Botrid团 \\ Faculty of Electrical Engineering, \\ Mechanical Engineering and Naval Architecture \\ University of Split, Boškovićeva b.b. 21000 Split \\ Croatia \\ (Dated: October 3, 2018)
}

\begin{abstract}
In contrast to the standard derivation of Kirchhoff's loop law, which invokes electric potential, we show, for the linear planar electric network in a stationary state at the fixed temperature, that loop law can be derived from the maximum entropy production principle. This means that the currents in network branches are distributed in such a way as to achieve the state of maximum entropy production.
\end{abstract}

PACS numbers: $05.70 . \mathrm{Ln}, 65.40 . \mathrm{Gr}$

Keywords: entropy production, mesh currents, Kirchhoff's laws

\section{INTRODUCTION}

Kirchhoff's laws 1] are the standard part of general physics courses [2, 3, 4]. In electrical engineering they are the starting point for the analysis of stationary processes in electric networks [5]. In the stationary state, due to the principle of charge conservation, Kirchhoff's current law (current law) is valid. It states that in each node of the network the sum of ingoing currents equals the sum of outgoing currents. Kirchhoff' loop law (loop law) is based on the assumption that electric potential is a well defined quantity in any point of the electric network. Then one can apply the principle of energy conservation to a macroscopic small amount of the charge circulating around the loop, i.e. the energy obtained on the sources should be equal to the dissipated energy. This statement is equivalent to the loop law, which states that the algebraic sum of electromotive forces (EMF-s) of the sources is equal to the sum of voltages (potential differences) in the loop. In this paper we show that the loop law can be derived for a linear planar network using the maximum entropy production principle [6]. This means that stationary state currents distribute themselves in the branches in such a way as to maximize the entropy production in the network.

\section{MESH CURRENTS AND LOOP LAW}

We consider a planar network (see Fig. 1). Assuming

\footnotetext{
*Electronic address: pasko@pmfst.hr

${ }^{\dagger}$ Electronic address: juretic@pmfst.hr

‡Electronic address: srecko.botric@fesb.hr
}

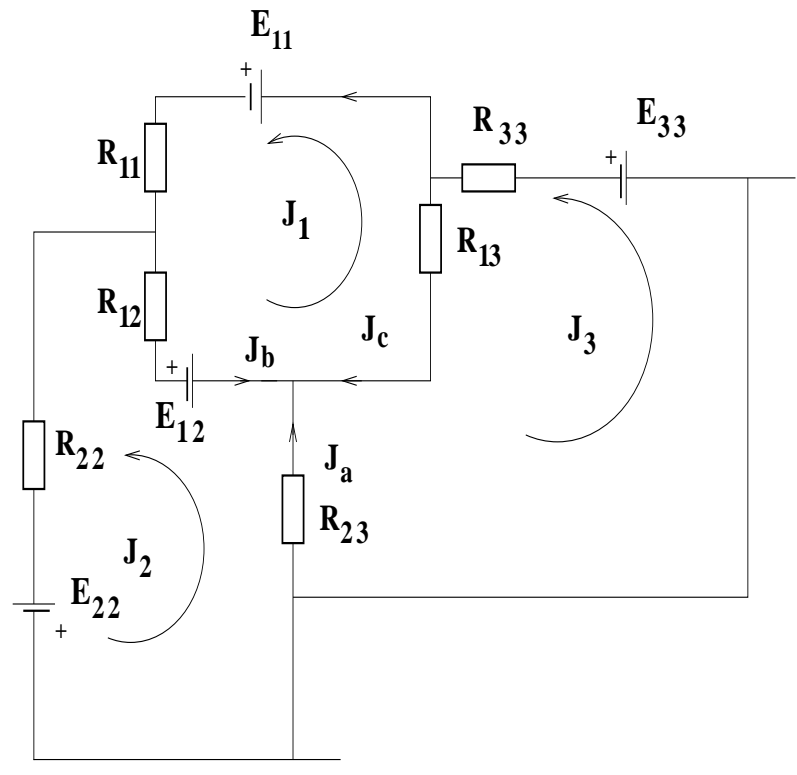

FIG. 1: A linear planar electric network

that network parameters, EMF-s and resistances, are fixed, one can find all currents applying current and loop law to nodes and loops. However, due to the current law, the currents in branches are not independent quantities.

Kirchhoff's laws give no prescription on how to find a set of independent currents for a given electric network. In the case of the planar network this problem has been solved by electrical engineers [5] by introducing the concept of mesh currents. Let us first define, within the network, the simple loop (mesh) as the one having no loop within it (loops 1,2 and 3 in Fig प). We associate a mesh current with each mesh $\left(J_{1}, J_{2}\right.$ and $J_{3}$ in Fig. 11). A current in a branch, common to two neighbor- 


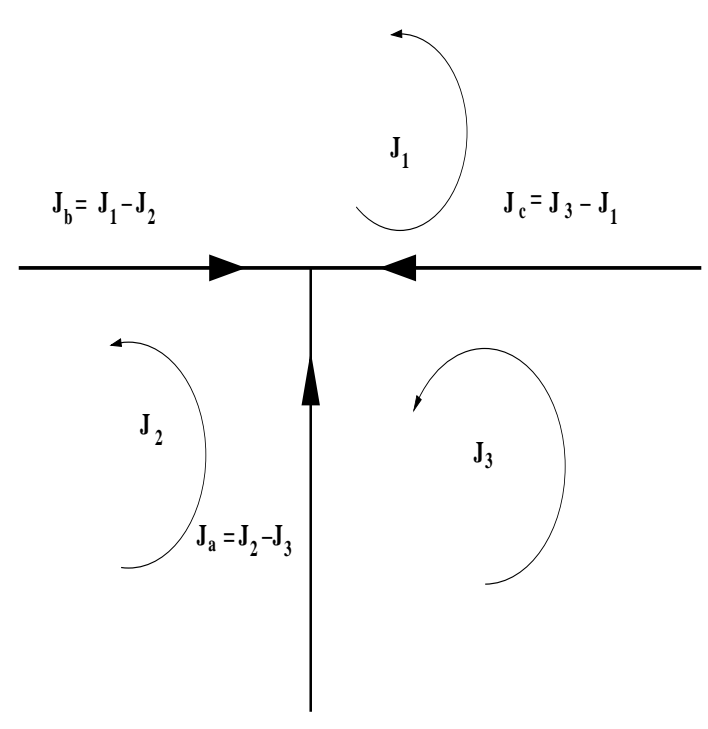

FIG. 2: Mesh currents and Kirchhoff's current law

ing meshes, is an algebraic sum of corresponding mesh currents (see Fig 2). The current in the outer branch, the branch which belongs to one mesh only, is equal to the mesh current. Evidently the mesh currents incorporate the current law (see Fig 2). It is easy to prove, by means of the mathematical induction, that the number of contour currents is equal to the number of independent currents in the network. The mesh currents are independent parameters determining the stationary state of the electric network as the thermodynamic system. In order to make the analysis of the network in terms of the mesh currents as simple as possible we introduce equivalent EMF-s and resistances. The equivalent EMF is equal to the algebraic sum of the EMF-s in a certain branch and the equivalent resistance is the sum of the resistances in that branch. We enumerate the meshes and corresponding mesh currents by single index notation, while the double index notations is used for the equivalent EMF-s and for equivalent resistances (Fig 1). Different indices in the double index notation appear when the single branch is shared between two meshes.

Applying the loop law for each mesh loop in terms of mesh currents we obtain the system of linear equations, the number of equations being equal to the number of mesh currents,

$$
\sum_{j} E_{i j}=R_{i i} J_{i}+\sum_{j} R_{i j}\left(J_{i}-J_{j}\right) .
$$

Note that the system of equations (1) incorporates both Kirchhoff's laws (due to the definition of the mesh currents). Here the left hand side of the equation is the algebraic sum of EMF-s within $i-t h$ mesh loop. The EMF is positive if mesh current comes out of its positive pole. Otherwise it is negative. Evidently it holds true

$$
E_{i j}=-E_{j i} \quad i \neq j
$$

and

$$
R_{i j}=R_{j i}
$$

We stress that the system of equations (1) assumes that the principle of energy conservation holds for each mesh separately (loop law). In the following we show that this system of equations (1) can be alternatively derived applying the maximum entropy production principle under the condition that the principle of energy conservation is valid for the whole network as the thermodynamic system.

\section{CONSERVATION OF THE ENERGY}

If the system at the fixed absolute temperature $T$ releases heat per unit of time, $d Q / d t$, the corresponding entropy production is

$$
\frac{d_{i} S}{d t}=\frac{1}{T} \frac{d Q}{d t}
$$

where index $i$, as it is introduced in the theory of non-equilibrium processes [7], emphasizes that only irreversible entropy change (rise) is considered.

The entropy production is the function of the thermodynamic state of a system. The stationary state of resistor at the fixed temperature is defined by the magnitude and the sense of the flow of the electric current. Due to the isotropy of the resistor the entropy production is invariant with respect to the flow direction of the current,

$$
\frac{d S_{i}}{d t}(-J)=\frac{d S_{i}}{d t}(J)
$$

Assuming that the stationary state of the resistor is close to its equilibrium state Taylor expansion of the entropy production gives,

$$
\frac{d S_{i}}{d t}=a+b J+c J^{2}+d J^{3}+\mathcal{O}\left(J^{4}\right) .
$$

A free term vanish since it corresponds to the entropy production in the equilibrium state. Due to the relation (5) coefficients of all odd powers vanish while $c>0$ due to $d S_{i} / d t>0$. Putting $c=R / T$, where $R$ is the resistance we get the well known expression for the dissipated electric power $d Q / d t=R J^{2}$. Evidently $c>0$ implies $R>0$.

A linear planar network can be separated into sources of the forces (EMF-s) and the passive part (resistors). In the stationary state there is no change in internal energy of the resistors while they convert energy from the sources into the heat given off to the surroundings. The rate of the energy conversion is given by,

$$
\frac{d Q}{d t}=\sum_{i} R_{i i} J_{i}^{2}+\frac{1}{2} \sum_{i j} R_{i j}\left(J_{i}-J_{j}\right)^{2} .
$$


Energy released by all sources (EMF-s) per unit of time is

$$
\frac{d W}{d t}=\sum_{i} E_{i i} J_{i}+\frac{1}{2} \sum_{i j} E_{i j}\left(J_{i}-J_{j}\right)
$$

The factor $\frac{1}{2}$, in these equations, appears due to the double counting in the case of internal branches.

Bearing in mind that resistors do not change their internal energy we can write, according to the first law of thermodynamics,

$$
\frac{d Q}{d t}-\frac{d W}{d t}=0
$$

i.e. taking into account (7) and (8) it holds,

$$
\begin{aligned}
& \sum_{i} E_{i i} J_{i}+\frac{1}{2} \sum_{i j} E_{i j}\left(J_{i}-J_{j}\right)= \\
= & \sum_{i} R_{i i} J_{i}^{2}+\frac{1}{2} \sum_{i j} R_{i j}\left(J_{i}-J_{j}\right)^{2} .
\end{aligned}
$$

\section{ENTROPY PRODUCTION AND ITS EXTREMUM IN THE ELECTRIC NETWORK}

We argue that Kirchhoff's loop law follows from the principle of the maximum overall entropy production in the network, assuming that the energy conservation (10) is satisfied. If there are $n$ meshes, and $n$ associated mesh currents, we have a conditional extremum problem in the $n$-dimensional linear space.

Assuming that all resistors in the network are at the same temperature $T$ the maximum of entropy production occurs at the same point of the $n$-dimensional space of currents as the maximum of generated heat (see eq. 44).

Standard procedure [8] of solving the conditional extremum introduces Lagrange's multipliers. In this case one seeks the extremum of the function

$$
F=\frac{d Q}{d t}+\lambda \Psi
$$

where due to the condition (10)

$$
\begin{gathered}
\Psi=\sum_{j} E_{j j} J_{j}+\frac{1}{2} \sum_{i, j} E_{i j}\left(J_{i}-J_{j}\right)- \\
-\left[\sum_{i} R_{i i} J_{i}^{2}+\frac{1}{2} \sum_{i, j} R_{i j}\left(J_{i}-J_{j}\right)^{2}\right]=0 .
\end{gathered}
$$

The function $F$ now reads

$$
\begin{aligned}
F=(1-\lambda) & {\left[\sum_{i} R_{i i} J_{i}^{2}+\frac{1}{2} \sum_{i, j} R_{i j}\left(J_{i}-J_{j}\right)^{2}\right]+} \\
+\lambda & {\left[\sum_{i} E_{i i} J_{i}+\frac{1}{2} \sum_{i, j} E_{i j}\left(J_{i}-J_{j}\right)\right] . }
\end{aligned}
$$

The solution of the system of $n$ equations

$$
\begin{array}{r}
\frac{\partial F}{\partial J_{i}}=2(1-\lambda)\left[R_{i i} J_{i}+\sum_{j} R_{i j}\left(J_{i}-J_{j}\right)\right]+ \\
+\lambda \sum_{j} E_{i j}=0,
\end{array}
$$

that satisfies the condition (12) is represented by the point in the $n$-dimensional liner space $\left\{J_{i}\right\}$ in which the function $d_{i} S / d t$ exhibits extremum. The value of Lagrange's multiplier $\lambda$ is uniquely determined by the system of equations (14) and eq. (12).

The standard, rather tedious, procedure of determining $\lambda$ can be avoided in the following way. Let us multiply eq. (14) with $J_{i}$ and sum over all mesh currents, i.e.

$$
\begin{array}{r}
2(1-\lambda)\left[\sum_{i} R_{i i} J_{i}^{2}+\sum_{i j} R_{i j}\left(J_{i}-J_{j}\right) J_{i}\right]+ \\
+\lambda \sum_{i j} E_{i j} J_{i}=0 .
\end{array}
$$

Due to the symmetry relations (21) and (3) we have,

$$
\sum_{i j} E_{i j} J_{i}=-\sum_{i j} E_{i j} J_{j}
$$

and

$$
\sum_{i j} R_{i j} J_{i}^{2}=\frac{1}{2} \sum_{i j} R_{i j}\left(J_{i}^{2}+J_{j}^{2}\right)
$$

By making use of eqs. (16) and (17) the eq. (15) becomes,

$$
\begin{array}{r}
2(1-\lambda)\left[\sum_{i} R_{i i} J_{i}^{2}+\frac{1}{2} \sum_{i j} R_{i j}\left(J_{i}-J_{j}\right)^{2}\right]+ \\
+\lambda\left[\sum_{i} E_{i i} J_{i}+\frac{1}{2} \sum_{i j} E_{i j}\left(J_{i}-J_{j}\right)\right]=0 .
\end{array}
$$

Substituting the term linear in mesh currents in eq. (18) by the term quadratic in mesh currents according to eq. (12) we get

$$
(2-\lambda)\left[\sum_{i} R_{i i} J_{i}^{2}+\frac{1}{2} \sum_{i j} R_{i j}\left(J_{i}-J_{j}\right)^{2}\right]=0,
$$

i.e. $\lambda=2$. One easily finds that the system of equations (14) for $\lambda=2$ is just the system of linear equations (11), which expresses the loop law.

Let us add that $F$ function for $\lambda=2$ is the $n$ dimensional paraboloid put upside-down, i.e. its extreme is maximum. In this way the equivalence between the maximum entropy production principle and Kirchhoff's loop law is established. In other words we can say that currents in a linear planar network distribute themselves so as to achieve the state of maximum entropy production. 


\section{DISCUSSION}

It was Ehrenfest (Enzykl. Math. Wissensch, IV, 2(II) fasc.6, p82, note23, 1912.) who first asked the question if there exists some function which, like the entropy in the equilibrium state of an isolated system, achieves its extreme value in the stationary non-equilibrium state.

The minimum entropy production theorem, attributed to Prigogine 7], identifies the entropy production due to irreversible processes in the system, as a physical function which becomes extremal in a stationary state. We agree with Jaynes opinion [9] that Prigogine's theorem is in fact the definition of the very special stationary state with zero induced flux, known as the static head state in electrical engineering [5] and in bioenergetics [10]. It is the quasi-equilibrium state that can be established close to equilibrium in the case of linear relationship between forces and fluxes, when induced force is left free to seek its maximal possible value. Energy conversion then stops at the level of driving force and corresponding flux, because induced flux vanishes and cannot be used to perform any work. One cannot obtain phenomenological equations, such as those found in references [14, 15] or eq. (1), from Prigogine's theorem, because that theorem does not have power of a principle. On the other hand phenomenological equations, such as eq. (1), can be derived from the maximum entropy production principle.

For the one-loop network the maximum entropy production principle holds as well. We leave for a reader to repeat our calculations from equations (11) to (19) in the case of the one-loop network. The claim that entropy production is minimal in the stationary state of the one-loop network [1] is wrong on two accounts. Firstly, authors 11] have presented potential drops as thermodynamical forces, i.e. they make no distinction between sources and loads and introduce artifical "forces" and "currents" in the entropy production expression. In the stationary state the charge conservation law requires that only one current flows in the one-loop network, which must be driven by single thermodynamic force (an algebraic sum of all EMF-s in the loop). Secondly, the statement about entropy production minimization in ref. [1] implies that minimum entropy production theorem [7] holds for a such network. This is not possible, because a minimum of two thermodynamic forces and corresponding currents and the condition of vanishing induced current (the static head non-equilibrium condition) must exist for the minimum entropy production theorem to hold.

An arbitrary network with no sources of electromotive forces (EMF) was first considered with respect to the entropy production by Kirchhoff 9] , Maxwell [12], and Jeans (see §357. in reference [13]). Kirchhoff postulated variational theorem 9], which states that in an arbitrary volume with fixed surface potential the currents distribute themselves so as to achieve the state of minimum entropy production. Since this example contains no sources of EMF, it represents a continuous extension of discrete network with no sources which was considered by Maxwell [12] and Jeans (see $\S 357$. in reference [13]). These authors came to the same conclusion that minimum entropy production governs current distribution, because they did not consider the energy conservation principle.

When EMF-s and the energy conservation principle are taken into account then the principle of the maximum overall entropy production in the network leads to Kirchhoffs loop law and accordingly to observed stationary current distribution as demonstrated in this paper. Let us note that Jeans considered an arbitrary, not only planar, electric network with EMF-s too. The maximum entropy production principle is implicitly present in his theorem (see $§ 358$. in reference 13$]$ ). This means that the current distribution in non-planar networks is also determined by the maximum entropy production principle. Jeans did not define independent currents in an arbitrary network, as we have done by introducing mesh currents in full agreement with charge conservation law. Therefore, it is relatively hard to follow his derivation.

Our macroscopic approach is very similar to that of Onsager who considered the related problem of heat conduction in an anisotropic crystal [14]. We shall show in a separate paper (in preparation) that an equivalence exists between the principle of least dissipation of energy [14, 15] and the maximum entropy production principle. Onsager was aware of the fact that the stationary state of heat conduction is in fact the state of maximum entropy production (see the sixth section in the reference 14]). The system of equations that describes stationary processes can be inferred from the maximum entropy production principle both in the case of heat conduction and in the case of linear planar electric network. The advantage of the maximum entropy production principle is that it offers a better physical insight through the explicit use of the energy conservation law in the conditional extremum problem. Rate of work done must be equal to heat produced in a stationary state. The dissipation function originally introduced by Lord Rayleigh [16] and also used by Onsager [14], obscures the distinction between energy sources and passive elements in the system, between the rate of work done and heat produced.

Beside macroscopic formulation of the maximum entropy production principle there exists the microscopic formulation too. It is due to Kohler 17] who demonstrated, starting from the Boltzmann transport equation, that fluxes in the stationary state of gases distribute themselves in order to achieve the state of maximum entropy production. Ziman 18 extended his work to free electron system in solids and demonstrated how this principle can be used in obtaining more accurate solutions of the Boltzmann transport equation. Recently, Dewar 6] has shown, applying Jaynes information theory formalism of statistical mechanics [19, 20], that stationary states are characterized by maximum entropy production.

To conclude, it is our belief that the principle of maximum entropy production is valid in linear non- 
equilibrium thermodynamics, at least for stationary processes. In other words we are convinced that it is just the principle Ehrenfest was looking for. We stressed in this paper that in each situation (linear planar electric network in this paper) this principle must be applied as the conditional extremum problem so that energy conservation law holds. Two of us have shown recently that biochemical cycle kinetics close to equilibrium state can be described by an analogue electrical circuit [21], and that modeling of photosynthesis 22] can also be done by using the maximum entropy production principle. These and other older [23] and more recent [6, 24] results using this principle show that the present derivation of Kirchhoff's loop law in this paper is likely to be only the beginning of the widespread use of this principle in many different scientific disciplines.

\section{Acknowledgments}

The present work is supported by Croatian Ministry of Science and Technology, project no. 0177163 to D.J.
[1] G. Kirchhoff, "Über die Auflösung der Gleichungen, auf welche man bei der Untersuchung der linearen Vertheilung galvanisher Ströme gefürt wird" Ann. Phys. und Chem., 72, 497, (1847).

[2] R.P. Feynman, R.B. Leighton and M. Sands, The Feynman Lectures on Physics, Vol. 2, (Addison-Wesley, Reading MA., 1964).

[3] F.W. Sears, M.W. Zemansky and H.D. Young, College physics, $7^{\text {th }}$ ed., (Addison-Wesley Publishing Company, Reading, Massachusetts, 1991).

[4] D. Halliday and R. Resnick, Fundamentals of Physics (John Wiley \& Sons, Inc., New York, 1988).

[5] D.E. Johnson, J.L. Hilburn and J.R. Johnson, Basic Electric Circuit Analysis (Prentice-Hall, New Jersey, 1986).

[6] R.C. Dewar, J. Phys. A. Math. Gen. 36, 631 (2003).

[7] I. Prigogine, Introduction to Thermodynamics of Irreversible Processes (Interscience, New York, 1967).

[8] M.I. Krasnov, G.I. Makarenko and A.I. Kiselev, Problems and Exercises in the Calculus of Variations (Mir Publishers, Moscow, 1975).

[9] E. T. Jaynes, Ann. Rev. Chem. 31, 579 (1980).

[10] S. R. Caplan and A. Essig, Bioenergetics and linear nonequilibrium thermodynamics (Harvard University Press, Cambridge, 1983).

[11] D. Kondepudi and I. Prigogine, Modern Thermodynamics (John Wiley \& Sons, Chichester, 2002).

[12] J. C. Maxwell, A Treatise on Electricity and Magnetism, Vol. 2, (Dover Publications, Inc., New York, 1954).

[13] J.H. Jeans, The Mathematical Theory of Electricity and
Magnetism (Cambridge at the University Press, Cambridge, 1923).

[14] L. Onsager, Phys. Rev. 37, 405 (1931).

[15] L. Onsager, Phys. Rev. 38, 2265 (1931).

[16] J.W.S. Rayleigh, The Theory of Sound, Vol. 1, (Dover Publications, Inc., New York, 1945).

[17] M. Kohler, Z. Physik, 124, 772 (1948).

[18] J. M. Ziman, Can J. Phys. 34, 1256 (1956).

[19] E.T. Jaynes, Phys. Rev. 106, 620 (1957).

[20] E.T. Jaynes, Phys. Rev. 108, 171 (1957).

[21] P. Županović and D. Juretić, Croat. Chem. Acta, 2004, in press.

[22] D. Juretić and P. Županović, Comput. Biol. Chem. 27, 541 (2003).

[23] A. Hill, Nature 348, 426 (1990); R. Robert and J. Sommeria, Phys. Rev. Lett. 69, 2776 (1992); P. Bak, C. Tang and K. Wiesenfeld, Phys. Rev. Lett. 59, 381 (1987); R. Swenson, Syst. Res. 6, 187 (1989); Y. Sawada, Prog. Theor. Phys. 66, 68 (1981); H. Ziegler, An Introduction to Thermomechanics, North-Holland, Amsterdam, 1983.

[24] R.D. Lorenz, J.I. Lunine, P.G. Withers and C.P. McKay, Geophys. Res. Lett. 28, 415 (2001); G.W. Paltridge, Q.J. Roy. Met. Soc. 127, 305 (2001); R. Lorenz, Science 299, 837 (2003); H.-J. Woo, Phys. Rev. E 66, 066104 (2002); H.-J. Woo, Europhys. Lett. 64, 627 (2003); R.C. Dewar, in Thermodynamics of Life, Earth and Beyond: Systems, Feedbacks, Organization, and Evolution, edited by A. Kleidon and R.D. Lorenz ( Springer Verlag, in press). 